\title{
Measurement of light absorbing particles in the snow of the Huaytapallana glacier in the central Andes of Peru and their effect on albedo and radiative forcing
}

\section{Medición de las partículas absorbentes de luz en la nieve del glaciar Huaytapallana en los Andes centrales de Perú y su efecto sobre el albedo y el forzamiento radiativo}

\author{
C. Torres ${ }^{1,2}$, L. Suárez ${ }^{1}$, C. Schmitt ${ }^{3}$, R. Estevan², D. Helmig ${ }^{4}$ \\ 1. Instituto Geofísico del Perú, Calle Badajoz 169, Ate, Lima, Perú \\ 2. Universidade Federal do Rio Grande, Avenida Itália km 8, Rio Grande, Rio Grande do Sul, Brasil \\ 3. National Center for Atmospheric Research, P.O. Box 3000, Boulder, Colorado, USA \\ 4. Institute of Arctic and Alpine Research, University of Colorado Boulder, Colorado, USA \\ ${ }^{(*)}$ E-mail: lsuarez@igp.gob.pe
}

Received: 18/06/2018 Accepted: 05/11/2018

DOI: $10.7149 /$ OPA.51.4.51004

\begin{abstract}
:
The impact of light absorbing particles (LAPs), i.e. black carbon (BC) and dust, in the cryosphere, is receiving increasing attention in the scientific community, because they may be contributing to the accelerating retreat of glaciers. The Huaytapallana mountain range in the central region of Peru is vulnerable to this effect; it has been estimated that its glacier surface has decreased by $56 \%$ in the last 27 years. In this study, we present first measurements of LAPs, in terms of effective black carbon $(\mathrm{eBC})$, in the surface snow (0-2 cm deep) in the Huaytapallana glacier between November 2015 and October 2016. The eBC mass was obtained using the new thermo-optical Light Absorption Heating Method (LAHM). The average of eBC in the surface layer was $31.1 \pm 22.2 \mathrm{ppb}$. Applying the Snow, Ice, and Aerosol Radiation (SNICAR) model we estimated a reduction in albedo from the $\mathrm{eBC}$ ranging from 0.6 to $5.0 \%$. The radiative forcing (RF) of the $\mathrm{eBC}$ was calculated, to be on average $11.8 \pm 7.8 \mathrm{~W} \mathrm{~m}^{-2}$. During winter and spring seasons, it further increased by $157 \%$ and $134 \%$, respectively, relative to the mean. Therefore, the impact of the eBC is an important factor in the retreat of the glacier Huaytapallana and must be considered in the hydrological prediction models of water availability.
\end{abstract}

Key words: effective black carbon, snow, radiative forcing.

\section{RESUMEN:}

El impacto de las partículas absorbentes de luz (PALs) como el carbono negro (CN) y el polvo en la criósfera está recibiendo creciente atención en la comunidad científica, debido a que estarían contribuyendo con el acelerado retroceso de los glaciares. La cordillera Huaytapallana en la región central del Perú, es vulnerable a este efecto, se ha estimado que su superficie glaciar disminuyó en $56 \%$ en los últimos 27 años. En este estudio, presentamos las primeras mediciones de las PALs, en términos de carbón negro efectivo ( $\mathrm{CNe}$ ) en la superficie de la nieve (0-2 cm de profundidad) en el glaciar Huaytapallana, entre noviembre de 2015 hasta octubre de 2016. La masa del CNe se determinó utilizando el nuevo Método termo-óptico de Calentamiento por Absorción de Luz (LAHM, por sus siglas en inglés). El promedio del CNe en la capa superficial fue $31.1 \pm 22.2 \mathrm{ppb}$. Aplicando el modelo de transferencia Radiativa de Nieve, Hielo y Aerosol (SNICAR, por sus siglas en inglés) estimamos una reducción del albedo debido al CNe en en un rango desde $0.6 \%$ a $5.0 \%$. El forzamiento radiativo (FR) del CNe fue calculado a ser en promedio $11.8 \pm 7.8 \mathrm{~W} \mathrm{~m}^{-2}$. Durante las estaciones de invierno y primavera, esto se incrementó en $157 \%$ y $134 \%$, respectivamente, en relación al promedio. Por lo tanto, el impacto del CNe es un factor importante en el retroceso del glaciar 
Huaytapallana y debe ser considerado en los modelos hidrológicos de predicción futura de disponibilidad de agua.

Palabras clave: carbón negro efectivo, nieve, forzamiento radiativo.

\section{REFERENCES AND LINKS / REFERENCIAS Y ENLACES}

[1] P. Krecl, A. C. Targino, L. Wiese, M. Ketzel, M. de Paula Corrêa, "Screening of short-lived climate pollutants in a street canyon in a mid-sized city in Brazil," Atmos. Pollut. Res., 7, 1022-1036 (2016).

[2] H. Niu et al., "In-situ measurements of light-absorbing impurities in snow of glacier on Mt. Yulong and implications for radiative forcing estimates," Sci. Total Environ., 581-582, 848-856 (2017).

[3] T. C. Bond et al., "Bounding the role of black carbon in the climate system: A scientific assessment," $J$. Geophys. Res. Atmos., 118, 5380-5552 (2013).

[4] T. H. Painter, M. G. Flanner, G. Kaser, B. Marzeion, R. A. VanCuren, W. Abdalati, "End of the Little Ice Age in the Alps forced by industrial black carbon.," Proc. Natl. Acad. Sci. U. S. A., 110, 15216-21 (2013).

[5] M. G. Flanner, C. S. Zender, J. T. Randerson, P. J. Rasch, "Present-day climate forcing and response from black carbon in snow," J. Geophys. Res., 112, (2007).

[6] J. Ming, C. Xiao, Z. Du, X. Yang, "An overview of black carbon deposition in High Asia glaciers and its impacts on radiation balance," Adv. Water Resour., 55, (2013).

[7] S. Yang, B. Xu, J. Cao, C. S. Zender, M. Wang, "Climate effect of black carbon aerosol in a Tibetan Plateau glacier," Atmos. Environ., 111, 71-78 (2015).

[8] K. M. Sterle, J. R. McConnell, J. Dozier, R. Edwards, M. G. Flanner, "Retention and radiative forcing of black carbon in eastern Sierra Nevada snow," Cryosph., 7, 365-374 (2013).

[9] T. J. Yasunari et al., "Estimated impact of black carbon deposition during pre-monsoon season from Nepal Climate Observatory - Pyramid data and snow albedo changes over Himalayan glaciers," Atmos. Chem. Phys., 10, 6603-6615 (2010).

[10] M. Dumont et al., "Contribution of light-absorbing impurities in snow to Greenland's darkening since 2009," Nat. Geosci., 7, 509-512 (2014).

[11] P. Chevallier, B. Pouyaud, W. Suarez, T. Condom, "Climate change threats to environment in the tropical Andes: glaciers and water resources," Reg. Environ. Chang., 11, 179-187 (2011).

[12] L. T. Molina et al., "Pollution and its Impacts on the South American Cryosphere," Earth's Futur., 3, 345369 (2015).

[13] T. P. Barnett, J. C. Adam, D. P. Lettenmaier, "Potential impacts of a warming climate on water availability in snow-dominated regions," Nature, 438, 303-309 (2005).

[14] B. G. Mark, G. O. Seltzer, "Evaluation of recent glacier recession in the Cordillera Blanca, Peru (AD 19621999): spatial distribution of mass loss and climatic forcing," Quat. Sci. Rev., 24, 2265-2280, (2005).

[15] J. T. Bury et al., "Glacier recession and human vulnerability in the Yanamarey watershed of the Cordillera Blanca, Peru," Clim. Change, 105, 179-206 (2011).

[16] W. Vergara et al., "Economic impacts of rapid glacier retreat in the Andes," Eos, Trans. Am. Geophys. Union, 88, 261-264 (2007).

[17] A. Rabatel et al., "Current state of glaciers in the tropical Andes: a multi-century perspective on glacier evolution and climate change," Cryosph., 7, 81-102 (2013).

[18] Y. Silva, K. Takahashi, R. Chávez, "Dry and wet rainy seasons in the Mantaro river basin (Central Peruvian Andes)," Adv. Geosci., 14, 261-264 (2008).

[19] J. I. López-Moreno et al., "Recent glacier retreat and climate trends in Cordillera Huaytapallana, Peru," Glob. Planet. Change, 112, 1-11 (2014.)

[20] M. 0. Andreae et al., "Carbon monoxide and related trace gases and aerosols over the Amazon Basin during the wet and dry seasons," Atmos. Chem. Phys., 12, 6041-6065 (2012).

[21] Q. Bourgeois, A. M. L. Ekman, R. Krejci, "Aerosol transport over the Andes from the Amazon Basin to the remote Pacific Ocean: A multiyear CALIOP assessment," J. Geophys. Res. Atmos., 120, 8411-8425 (2015).

[22] A. S. Moya Álvarez, R. E. Arredondo, R. Á. Yuli Posadas, "Determinación de la presencia de partículas (PM10) en Perú producidas por quema de biomasa con ayuda de modelos numéricos," Rev. Int. Contam. 
Ambient., 33,. 99-108 (2017).

[23] ANA, "Inventario de Glaciares y Lagunas," 2014. [Online]. Available: http://www.ana.gob.pe/media/981508/glaciares.pdf.

[24] C. G. Schmitt et al., "Measurements of light-absorbing particles on the glaciers in the Cordillera Blanca, Peru," Cryosph., 9, 331-340 (2015).

[25] W. J. Wiscombe, S. G. Warren, W. J. Wiscombe, S. G. Warren, "A Model for the Spectral Albedo of Snow. I: Pure Snow," J. Atmos. Sci., 37, 2712-2733 (1980).

[26] O. B. Toon, C. P. McKay, T. P. Ackerman, K. Santhanam, "Rapid calculation of radiative heating rates and photodissociation rates in inhomogeneous multiple scattering atmospheres," J. Geophys. Res., 94, 16287 (1989).

[27] S. Platnick, L. Oreopoulos, "Radiative susceptibility of cloudy atmospheres to droplet number perturbations: 1. Theoretical analysis and examples from MODIS," J. Geophys. Res., 113, D14S20, (2008).

[28] I. Reda, A. Andreas, "Solar position algorithm for solar radiation applications," Sol. Energy, 76, 577-589 (2004).

[29] T. H. Painter, A. C. Bryant, S. M. Skiles, "Radiative forcing by light absorbing impurities in snow from MODIS surface reflectance data," Geophys. Res. Lett., 39, (2012).

[30] S. Kaspari, T. H. Painter, M. Gysel, S. M. Skiles, M. Schwikowski, "Seasonal and elevational variations of black carbon and dust in snow and ice in the Solu-Khumbu, Nepal and estimated radiative forcings," Atmos. Chem. Phys., 14, 8089-8103 (2014).

[31] R. L. Armstrong, E. Brun, Snow and Climate: Physical Processes, Surface Energy Exchange and Modeling, vol. 1. (2008).

[32] G. Trasmonte, Y. Silva, R. Chavez, B. Segura, "Trends in maximum and minimum temperature in the central Andes of Peru (Mantaro river basin)," Actas la Octava "International Conf. South. Hemisph. Meteorol. Oceanogr., 463-468 (2006).

[33] W. Suarez et al., "Balance enérgetico neto (2012-2014) y evolución temporal del nevado Quisoquipina en la región de Cusco (1990-2010)," Rev. Peru. GEO-ATMOSFÉRICA, 4, 85-92, (2015).

[34] J. E. Sicart, P. Wagnon, P. Ribstein, "Atmospheric controls of the heat balance of Zongo Glacier $\left(16^{\circ} \mathrm{S}\right.$, Bolivia)," J. Geophys. Res., 110, D12106, (2005).

[35] B. K. Veettil, S. Wang, S. Florêncio de Souza, U. F. Bremer, J. C. Simões, "Glacier monitoring and glacierclimate interactions in the tropical Andes: A review," J. South Am. Earth Sci., 77, 218-246 (2017).

[36] J.-G. Winther, O. Bruland, K. Sand, Å. Killingtveit, D. Marechal, "Snow accumulation distribution on Spitsbergen, Svalbard, in 1997," Polar Res., 17, 155-164 (1998).

[37] S. G. Warren et al., "Snow Depth on Arctic Sea Ice," J. Clim., 12, 1814-1829 (1999).

[38] I. Sobota, "Snow accumulation, melt, mass loss, and the near-surface ice temperature structure of Irenebreen, Svalbard," Polar Sci., 5, 327-336 (2011).

[39] S. J. Doherty, C. Dang, D. A. Hegg, R. Zhang, S. G. Warren, "Black carbon and other light-absorbing particles in snow of central North America," J. Geophys. Res. Atmos., 119, 12,807-12,831 (2014).

[40] X. Wang, S. J. Doherty, J. Huang, "Black carbon and other light-absorbing impurities in snow across Northern China," J. Geophys. Res. Atmos., 118, 1471-1492 (2013).

[41] S. S. de Vasconcelos, P. M. Fearnside, P. M. L. de A. Graça, D. V. Dias, F. W. S. Correia, "Variability of vegetation fires with rain and deforestation in Brazil's state of Amazonas," Remote Sens. Environ., 136, 199-209 (2013).

[42] H.-W. Jacobi et al., "Black carbon in snow in the upper Himalayan Khumbu Valley, Nepal: observations and modeling of the impact on snow albedo, melting, and radiative forcing," Cryosph., 9, 1685-1699 (2015).

[43] Y. Zhang et al., "Light-absorbing impurities enhance glacier albedo reduction in the southeastern Tibetan plateau," J. Geophys. Res. Atmos., 122, 6915-6933 (2017). 


\section{Introducción}

Los Contaminantes Climáticos de Vida Corta (CCVC) han atraído la atención de la comunidad científica debido al importante rol que juegan en el sistema climático de la Tierra. Estos contaminantes tienen un tiempo de residencia relativamente corta en la atmósfera, por lo que representa un componente importante para mitigar el calentamiento global. Los principales CCVC son partículas de carbono negro (CN), ozono troposférico $\left(\mathrm{O}_{3}\right)$ y metano $\left(\mathrm{CH}_{4}\right)$ [1]. El CN es un tipo distinto de material carbonáceo, que se genera en la combustión incompleta de la quema de biomasa y los combustibles fósiles [2].

El CN afecta principalmente al clima de dos maneras. Por un lado, al estar suspendido en la atmósfera produce un forzamiento radiativo (FR) positivo debido a que absorbe fuertemente la luz visible. Bond et al. (2013) evaluaron el FR del CN en la atmósfera, estimando un valor de $0.71 \mathrm{~W} \mathrm{~m}^{-2}$ con un nivel de incertidumbre del 90\%. Por otro lado, cuando se deposita sobre la nieve y el hielo, reduce su albedo provocando mayor retención de energía y por lo tanto mayor fusión. Painter et al. (2013) informaron que los glaciares de los Alpes debieron haber seguido creciendo hasta el año 1910 de acuerdo con los registros de temperatura y precipitación, pero estos comenzaron a retroceder abruptamente en el año 1850, la deposición del CN en la nieve pudo haber sido el motor de este abrupto retroceso debido a que el FR aumentó entre los años de 1850 y 1990, así como también la masa del CN aumentó de acuerdo con los análisis de los núcleos de hielo. Los estudios de Flanner et al. (2007); Ming et al. (2013); Yang et al. (2015); y Niu et al. (2017) evaluaron el FR del CN en los glaciares de la Meseta Tibetana, donde encontraron valores entre 76-146 W m-2. En el estudio de Sterle et al. (2013) evaluaron el FR del CN en América del Norte, donde encontraron valores entre 20-40 $\mathrm{W} \mathrm{m}^{-2}$. El estudio de Yasunari et al. (2010) evaluaron a partir de experimentos numéricos simples que las reducciones del albedo entre $2.0-5.2 \%$ en los glaciares del Himalaya conducirían a un aumento de la escorrentía entre 70-204 mm. En el estudio de Dumont et al. (2014) estimaron que una disminución en el albedo de la nieve del 1\% en Groenlandia conduciría a una pérdida de masa superficial de $27 \mathrm{Gt}$ año-1. Estos resultados indican que el oscurecimiento de la nieve es un componente importante. Por lo tanto, el impacto de la deposición del CN en la nieve y el hielo no debe ser ignorado en las predicciones futuras de disponibilidad de agua.

La cordillera de los Andes se extiende por toda la parte occidental de América del Sur, albergando el 99\% de los glaciares tropicales del mundo, de los cuales el 71\% se encuentran en Perú [11], [12]. Los principales ríos en esta región se forman en las cabeceras de las cuencas hidrográficas que están ubicadas en los Andes, como resultado de la precipitación y el deshielo de los glaciares [13]. Los glaciares son una fuente importante de agua en temporada seca, que sirve para el riego de la agricultura tradicional en las montañas, la agricultura intensiva moderna en las estribaciones y en tierras bajas [14], [15], y así como para la producción de energía mediante hidroeléctricas [11], [16]. Los glaciares Andinos han retrocedido a un ritmo acelerado en las últimas décadas [17]. Este retroceso acelerado tendrá implicaciones potencialmente grandes en la disponibilidad de los agua para las poblaciones ubicadas en esta región en el futuro.

El deshielo de los glaciares ubicados en la cordillera Huaytapallana abastece a las subcuencas de los ríos Shullcas y Achamayo que son parte del valle del Mantaro. Esta región juega un papel importante en la economía nacional. La producción agrícola en el valle proporciona alimentos significativos para el mercado interno de Lima [18]. Además, contribuye con el abastecimiento del agua para el consumo humano de las ciudades de Concepción y Huancayo donde se concentra la mayor parte de la población urbana $(\sim 470000$ habitantes). El agua que no se utiliza desemboca a la cuenca del río Mantaro que es una fuente importante de generación hidroeléctrica, la cual suministra el $\sim 35 \%$ de la capacidad nacional [18]. Según el estudio de López-Moreno et al. (2014), en esta región la temperatura máxima se incrementó significativamente con una tasa de calentamiento de $0.22^{\circ} \mathrm{C}$ década $^{-1}$ al igual que la precipitación, como consecuencia elárea glacial se redujo en 55\% entre los años 1984 y 2011. Sin embargo, es necesario evaluar otros factores que podrían estar contribuyendo con el acelerado retroceso de estos glaciares, debido a que no es posible explicar este abrupto retroceso atribuyendo solamente al efecto de la temperatura y la precipitación. Por ello, en este estudio se caracteriza la variación temporal de las partículas absorbentes de luz (PALs) depositadas en la nieve y se estima el efecto sobre el albedo y el FR, como consecuencia del oscurecimiento de la nieve en el glaciar Huaytapallana. 


\section{Materiales y métodos}

\section{2.a. Descripción del sitio}

El área del estudio es el glaciar Huaytapallana ubicado en la cordillera Huaytapallana el cual se encuentra en los Andes centrales de Perú (lat. $-11.95^{\circ}$ a $-11.83^{\circ}$; lon. $-75.01^{\circ}$ a $-74.99^{\circ}$ ). Hacia el este se ubica la región amazónica de Brasil y Perú, y al sureste Bolivia (figura 1a). Estas regiones se ven afectadas por la ocurrencia de un gran número incendios de vegetación abierta. Las emisiones de estas regiones se transportan a los Andes [20]-[22].

El deshielo de los glaciares de la cordillera Huaytapallana contribuye con la escorrentía de los ríos Shullcas y Achamayo. Las ciudades de Concepción y Huancayo usan esta agua para consumo humano, ganadería, agricultura y actividades económicas (figura 1b). El agua que no se usa desemboca al río Mantaro, que forma parte de la cuenca del Amazonas.

La cordillera Huaytapallana contiene 105 glaciares que se extienden desde 4900 hasta 5527 m.s.n.m. con un área de $26.4 \mathrm{Km}^{2}$ [23]. Los glaciares son típicamente de tipo circo y muchos son glaciares colgantes y muy abruptos debido a la pendiente. Esta región también tiene numerosos lagos, muchos de los cuales se formaron en las últimas décadas debido al rápido retroceso de los glaciares en esta región [19].

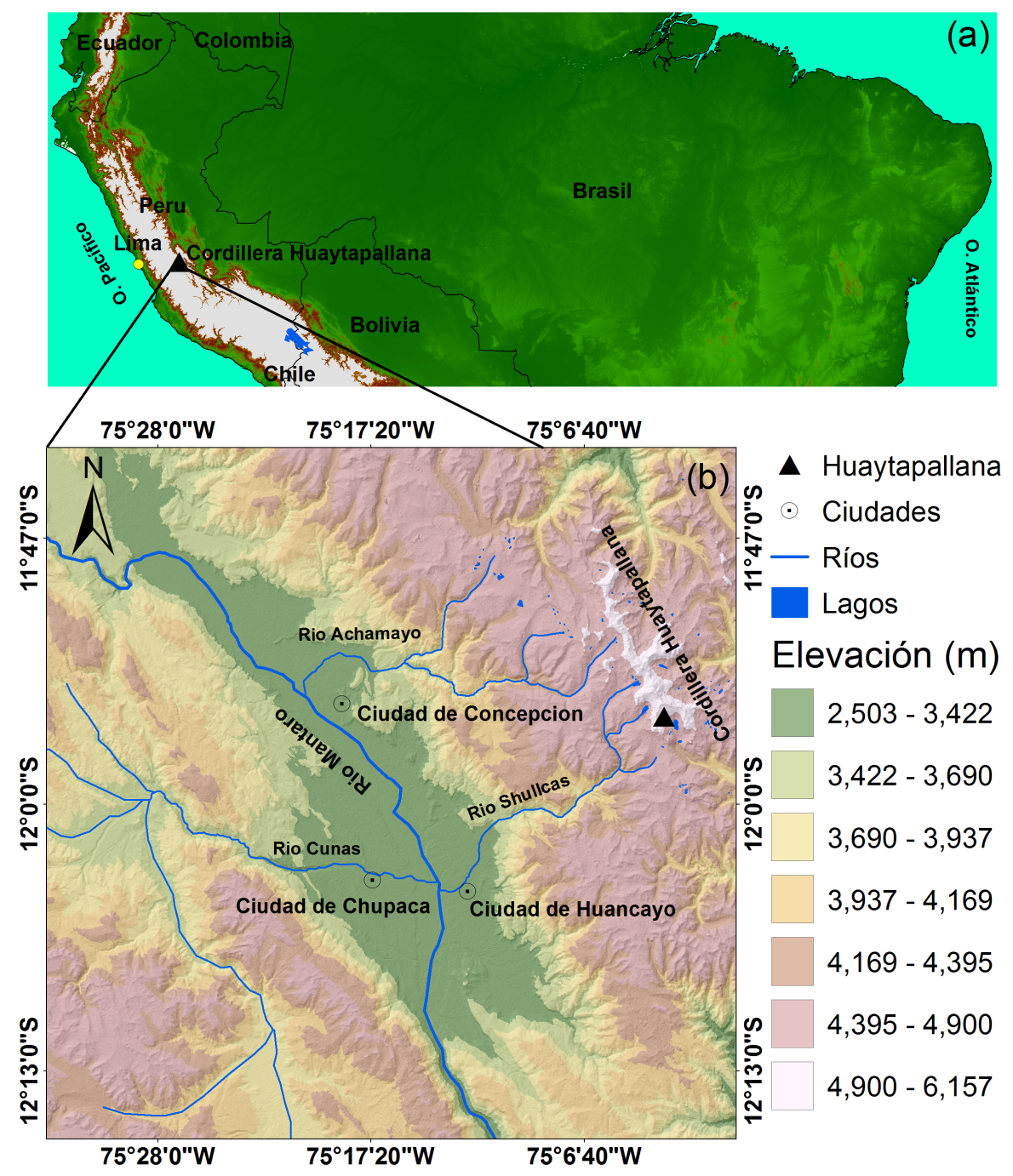

Fig.1. Ubicación de la Cordillera Huaytapallana (a) escala regional y (b) escala local. 


\section{2.b. Recolección de datos}

\section{2.b.1. Datos meteorológicos}

La información de las condiciones meteorológicas diarias, como la temperatura del aire, la precipitación y la humedad relativa, se obtuvieron de la estación meteorológica Huaytapallana (lat. $-11.93^{\circ}$; lon. $-7^{75.06^{\circ}}$; ele. 4684 m.s.n.m.) el más cercano al sitio de estudios. Esta estación es administrada por el Servicio Nacional de Meteorología e Hidrología (SENAMHI) del Perú. Para medir la temperatura del aire (precisión $\pm 0.5^{\circ} \mathrm{C}$ a $-40{ }^{\circ} \mathrm{C}$ ) y la humedad relativa (precisión $\pm 1 \%$ a $20^{\circ} \mathrm{C}$ ) la estación está equipada con un sensor Campbell Scientific HMP45C-L, y para la precipitación (precisión $\pm 2 \%$ a $<250 \mathrm{~mm} \mathrm{~h}^{-1}$ ) un sensor Campbell Scientific TB4-L. Los datos se almacenan en el registrador CR1000 y se transmiten a través de una antena GOES 25316. Los datos pueden ser observados en tiempo real en la plataforma del SENAMHI en el siguiente enlace http://www.senamhi.gob.pe/include_mapas/_dat_esta_tipo.php?estaciones=472DF5D6.

La estación meteorológica Huaytapallana no registra datos de radiación solar de la superficie de la tierra. Por ello, recurrimos al Sistema de Energía Radiante y de Nubes de la Tierra (CERES, por sus siglas en inglés) de la Administración Nacional de la Atmósfera y el Espacio (NASA, por sus siglas en inglés) de los Estados Unidos (EE. UU.). El producto CERES SYNI1 deg contiene datos de radiancia de la superficie de la Tierra. Para generar esta colección de datos primero obtienen las propiedades ópticas de las nubes y los aerosoles, principalmente del Espectroradiómetro de Imágenes de Resolución Moderada (MODIS, por sus siglas en inglés) a bordo de los satélites TERRA y AQUA. Para los aerosoles, además de los datos de MODIS se complementan con datos de asimilación provenientes del Modelo de Transporte Atmosférico y Química (MATCH, por sus siglas en inglés) para mejorar la resolución temporal. Todos estos datos se ingresan al modelo de trasferencia radiativa Langley Fu-Liou para producir flujos de radiación solar incidente y reflejada de onda larga (LW, por sus siglas en inglés) y corta (SW, por sus siglas en inglés) para condiciones de cielo claro (sin nubes) y todas las condiciones de cielo (con nubes y aerosoles). Luego, los datos generados por el modelo se comparan con observaciones en tierra y se ponen a disposición de la comunidad científica en la plataforma de CERES en el siguiente enlace https://ceres-tool.larc.nasa.gov/ordtool/jsp/SYN1degSelection.jsp, a una resolución espacial de $1^{\circ}$ y temporal promedio cada 3 horas, diaria y mensual.

El satélite TERRA tiene una órbita casi polar sincronizada con el sol, pero con un tiempo de cruce ecuatorial de aproximadamente 10:30 tiempo local (TL) y 22:30 TL. El sensor AQUA también tiene una órbita casi polar sincrónica con el sol, pero cruza el ecuador a las 13:30 TL y a las 02:30 TL.

El conjunto de datos CERES utilizado en este estudio incluye la radiación solar de SW (0.3 a $5 \mu$ m región espectral) para todas las condiciones de cielo desde noviembre de 2015 hasta octubre de 2016, con resolución temporal promedio de 3 horas $(1: 30,4: 30,7: 30,10: 30,13: 30,16: 30,19: 30,22: 30$ UTC) y la ubicación del píxel latitud $-11.5^{\circ}$ y longitud $-75.5^{\circ}$.

\section{2.b.2. Datos de CNe y propiedades físicas de la nieve}

Para determinar la masa de las PALs depositadas en la nieve del glaciar Huaytapallana se realizaron expediciones de campo al glaciar desde noviembre de 2015 hasta octubre de 2016 (una vez por mes). Se recolectaron muestras de nieve en bolsas plásticas de ziplock, para ello se identificaron entre 2 y 3 puntos de muestreo, recolectando aproximadamente $1 \mathrm{~kg}$ de nieve de la capa superficial $(0-2 \mathrm{~cm}$ de profundidad). Los puntos de muestro se fijaron lo más alto posible y alejados de superficies sin glaciar para evitar el polvo, dependiendo siempre de la accesibilidad a los sitios. En el campamento las muestras de nieve se derritieron una por una, para ello las bolsas de ziplock se colocaron en agua tibia a una temperatura aproximada de 30 ${ }^{\circ} \mathrm{C}$. Una vez fundida, el agua de nieve se extrajo en jeringas de $60 \mathrm{ml}$ y se bombeó a través del filtro de fibra de cuarzo con porosidad de $0.7 \mu \mathrm{m}$ y diámetro de $25 \mathrm{~mm}$ del tipo "Pallflex Tissuquartz". Se filtraron un total de $600 \mathrm{ml}$ de agua por muestra a menos que el filtro se obstruye demasiado, en cuyo caso, se registró la cantidad de agua filtrada. Los filtros son retirados del soporte, se colocan en capsulas de plástico y se mantienen en su lugar con un anillo de espuma. Después de cada campaña de campo los filtros son almacenados en un congelador hasta realizar 3 campañas para ser enviados a los EE. UU. Una vez que los filtros llegan a los EE. UU. son analizados con un nuevo Método termo-óptico de Calentamiento por Absorción de Luz (LAHM, por sus siglas en inglés), la premisa de este método se discute en Schmitt et al. (2015). El método LAHM incluye también el impacto del polvo en la absorción de la radiación solar. El polvo presente en la nieve puede ser de origen humano (prácticas agrícolas y carreteras sin asfalto). Por ello en este estudio se usa el término de carbón negro efectivo (CNe) para reportar los valores de las PALs. 
Paralelamente al muestreo de nieve también se realizaron mediciones de la densidad de la nieve $\left(\rho_{\text {nieve }}\right)$. Para ello, se utilizó una técnica tradicional que consiste en introducir un tubo de acero inoxidable (diámetro $=6 \mathrm{~cm}$ y altura $=11$ ) en la capa de nieve en cada punto de muestreo seleccionado para luego medir la masa con una balanza mecánica colgante de $5 \mathrm{~kg}$. Para el radio efectivo del grano de la nieve $\left(R_{\text {nieve }}\right)$, se consideró el valor de $250 \mu \mathrm{m}$ para todos los casos de modelado, que es el considerado para nieve reciente de grano fino [6].

\section{2.c. Descripción del modelo SNICAR}

Para estimar el impacto de la deposición del CNe en el albedo de la nieve se empleó el modelo de transferencia Radiativo de Nieve, Hielo y Aerosol (SNICAR, por sus siglas en inglés) de Flanner et al. (2007). El modelo SNICAR utiliza la teoría de Wiscombe et al. (1980) y la aproximación de dos corrientes propuesta por Toon et al. (1989) para resolver la ecuación de transferencia radiativa. Los parámetros de entrada al modelo SNICAR son directamente observables como: el tipo de la radiación solar incidente (directa o difusa), el ángulo cenital solar (SZA, por sus siglas en ingles) en caso de que el tipo de la radiación solar sea directa, $R_{\text {nieve }}, \rho_{\text {nieve }}$, la profundidad de la capa de nieve, el albedo de banda ancha de la superficie subyacente y las impurezas presentes en la nieve (CN, polvo y ceniza volcánica). A continuación, se describen algunos de estos parámetros debido a que ya el resto fueron descritos en la sección 2.2.2.

\section{2.d. Parámetros de entrada al modelo SNICAR}

\section{2.d.1. El tipo de la radiación solar incidente}

Este parámetro determina el tipo de la radiación solar que incide sobre la superficie del glaciar, directa o difusa. Los factores más importantes que determina este parámetro son los aerosoles y las nubes. En este estudio se realizó un análisis de las propiedades ópticas de la nube para un radio de $10 \mathrm{~km}$, tomando como punto centro el sitio de muestreo (lat. $-11.94^{\circ}$; lon. $-75.03^{\circ}$ ). Para ello, se utilizó el producto MOD06 L2 del sensor MODIS a bordo del satélite TERRA para las 10:30 TL de cruce ecuatorial para los días de campaña. Se extrajeron los valores de los parámetros ópticos de las nubes como: la fase y el espesor óptico. La fase de la nube determina el tipo de partículas que conforman la nube, o sea, si es líquida o hielo. Con el espesor óptico de la nube (COT, por sus siglas en inglés) se calcula la transmitancia total $\left(T_{\text {total }}\right)$ de la nube (Eq. 1) propuesta por Platnick y Oreopoulos (2008).

$$
T_{\text {total }}=\frac{2}{(2+(1-g) \times C O T)}
$$

Donde, $g$ es el parámetro de asimetría de las partículas en la nube $(g=0.85$ para nubes de agua líquida y $g=0.76$ para nubes de hielo). $T_{\text {total }}$ considera las componentes de transmitancia de la nube, tanto directa $\left(T_{\text {directa }}\right)$ como difusa $\left(T_{\text {difusa }}\right)$. Para separar estas componentes se aplican las ecuaciones 2 y 3 (Platnick, comunicación personal).

$$
\begin{gathered}
T_{\text {directa }}=e^{\left(\frac{-C O T}{\mu_{0}}\right)} \\
T_{\text {difusa }}=T_{\text {total }}-T_{\text {directa }}
\end{gathered}
$$

Donde, $\mu_{0}$ es SZA. La clasificación del tipo de la radiación solar para el modelado en este estudio se determinó en base a las siguientes condiciones: si $T_{\text {directa }}>T_{\text {difusa }}, \rightarrow$ radiación indecente $=$ directa y si $T_{\text {directa }}<T_{\text {difusa }}, \rightarrow$ radiación indecente $=$ difusa. En este caso tanto $T_{\text {directa }}$ como $T_{\text {difusa }}$ fueron el promedio para el radio de $10 \mathrm{~km}$.

\section{2.d.2. El SZA}

Este parámetro determina la posición del sol sobre la vertical. Los principales factores que influyen en su variación son el tiempo y la ubicación geográfica (latitud, longitud y altitud). El SZA define en gran medida la intensidad de la radiación solar en cada región, siendo inversamente proporcional. En este estudio se empleó el algoritmo de posición solar de Reda y Andreas (2004). Este algoritmo considera todos los parámetros astronómicos para su cálculo. Se utilizó este algoritmo debido a la incertidumbre muy baja de cálculo de los ángulos cenital y azimutal del sol, la incertidumbre es de $\pm 0.0003^{\circ}$ para periodos entre los años 2000 y 6000. 
Calculamos el SZA para todos los días de campaña de muestreo a un minuto de resolución temporal para la siguiente ubicación geográfica: latitud $-11.95^{\circ}$, longitud $-75.03^{\circ}$ y elevación 5100 m.s.n.m. que corresponde al sitio de muestreo de nieve.

TABLA 1. Algunos parámetros de entrada al modelo SNICAR y la masa del CNe.

\begin{tabular}{clllll}
\hline Fecha & $\begin{array}{c}\text { Tipo de } \\
\text { Radiación }\end{array}$ & $\begin{array}{c}\text { SZA } \\
\left({ }^{\circ} \mathbf{)}\right.\end{array}$ & $\begin{array}{c}\boldsymbol{\rho}_{\text {nieve }} \\
\left(\mathbf{k g ~ m}^{-3} \mathbf{)}\right.\end{array}$ & $\begin{array}{c}\boldsymbol{R}_{\text {nieve }} \\
(\boldsymbol{\mu m})\end{array}$ & $\begin{array}{c}\mathbf{C N e} \\
(\mathbf{p p b})\end{array}$ \\
\hline $2015-11-22$ & Difusa & 9.1 & 329 & 250 & 25.3 \\
$2015-12-20$ & Directa & 13.2 & 337 & 250 & 10.0 \\
$2016-01-17$ & Difusa & 13.0 & 358 & 250 & 10.8 \\
$2016-02-21$ & Directa & 10.8 & 353 & 250 & 3.9 \\
$2016-03-20$ & Difusa & 15.3 & 353 & 250 & 43.2 \\
$2016-04-17$ & Difusa & 23.9 & 334 & 250 & 8.7 \\
$2016-05-22$ & Difusa & 33.1 & 324 & 250 & 29.0 \\
$2016-06-29$ & Directa & 36.1 & 316 & 250 & 20.9 \\
$2016-07-17$ & Directa & 34.1 & 319 & 250 & 83.9 \\
$2016-08-21$ & Difusa & 25.1 & 317 & 250 & 46.8 \\
$2016-09-25$ & Difusa & 11.9 & 320 & 250 & 51.5 \\
$2016-10-23$ & Directa & 3.5 & 324 & 250 & 38.4 \\
\hline
\end{tabular}

\section{2.e. Estimación del FR}

Para estimar el FR como consecuencia de la deposición del CNe se utilizó el modelo SNICAR [5], [25], [26] para evaluar el albedo de la nieve en condiciones de nieve limpia y con impurezas ( $\mathrm{CNe}$ ). Al integrar la diferencia del albedo de nieve limpia y con impurezas, con la radiancia espectral, se obtiene el FR (Eq. 4) [2], [7], [8], [29], [30].

$$
F R=\sum_{0.305 \mu m}^{4.995 \mu m} E \downarrow(\lambda, \theta) \times\left(\alpha_{\text {lim. }}\left(R_{\text {nieve }}, \lambda\right)-\alpha_{\text {imp. } .(C N)}\left(R_{\text {nieve }}, \lambda\right)\right) \times \Delta \lambda
$$

Donde, $E \downarrow$ es la radiancia espectral incidente proporcionada por CERES, para este estudio se extrajeron los valores de las 11:30 TL, $\alpha_{l i m}$. es el albedo limpio y $\alpha_{i m p .(C N)}$ es el albedo con impurezas, en este caso solo para $\mathrm{CNe}$, ambos proporcionados por el modelo SNICAR. El modelo SNICAR se configura de la siguiente manera: el método de aproximación de dos corrientes fue Hemispheric Mean porque las aproximaciones Delta-Eddington y Quadrature pueden proporcionar albedo negativo en el IR cercano en condiciones de luz difusa [26], en este estudio 7 expediciones de campo fueron determinados para condiciones difusa; el albedo de banda ancha de la superficie subyacente fue 0.53 considerado para una superficie de hielo [31]; el número de capas de nieve fueron $4(0,2,10,20,30 \mathrm{~cm})$ en la primera capa se consideró la masa medida del CNe. En la tabla 1 se observa algunos de los parámetros de entrada al modelo SNICAR.

\section{Resultado y discusión}

\section{3.a. Descripción meteorología}

La variación temporal de la temperatura del aire ( $2 \mathrm{~m}$ del suelo) para el periodo de estudios se representa en la figura $2 \mathrm{a}$. La temperatura promedio mensual fue $3.5 \pm 0.7^{\circ} \mathrm{C}(\bar{x} ; \sigma)$. La mayor temperatura fue registrada en enero $\left(4.4^{\circ} \mathrm{C}\right)$, mientras que el mes con menor temperatura fue agosto $\left(2.4^{\circ} \mathrm{C}\right)$. Esta variación es típica de la región tropical andina como lo describen Trasmonte et al. (2006). La variación temporal de la precipitación se representa en la figura $2 \mathrm{~b}$ con un acumulado para el periodo de $771.1 \mathrm{~mm}$ año-1. El mes 
con mayor precipitación fue febrero (207.4 $\mathrm{mm} \mathrm{mes}^{-1}$ ), mientras que el mes con menor precipitación fue julio (4.3 $\mathrm{mm} \mathrm{mes}^{-1}$ ). Esta variación es también típica de esta región como lo describen Silva et al. (2008). La variación temporal de la humedad relativa se representa en la figura 2c. La humedad promedio mensual fue $67.5 \pm 9.2 \%$. Como era de suponer el mes con mayor humedad fue registrado en febrero (80.7\%), mientras que el mes con menor humedad fue en julio (50.5\%). La variación temporal de la radiación solar incidente de SW se representa en la figura 2d. El promedio para todo el periodo de estudios fue 218.5 \pm 11.0

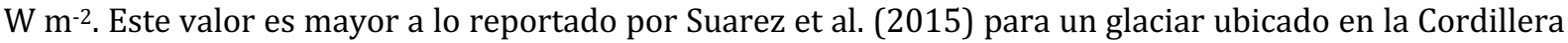
Quisoquipina (lat. $-13.80^{\circ}$; lon. $-70.74^{\circ}$; ele. 5180 m.s.n.m.) con una media anual de $187.5 \pm 6.4 \mathrm{~W} \mathrm{~m}^{-2}$, probablemente esto se deba a la presencia del fenómeno del Niño durante el año 2016, que en consecuencia provoca la disminución de la nubosidad y las lluvias en la región central y sur de los Andes. La variación del promedio mensual de la radiación solar que incide en la superficie del glaciar es bastante constante durante todo el año, como lo reporta Sicart et al. (2005) para el glaciar Zongo localizado en la Cordillera Real de Bolivia.
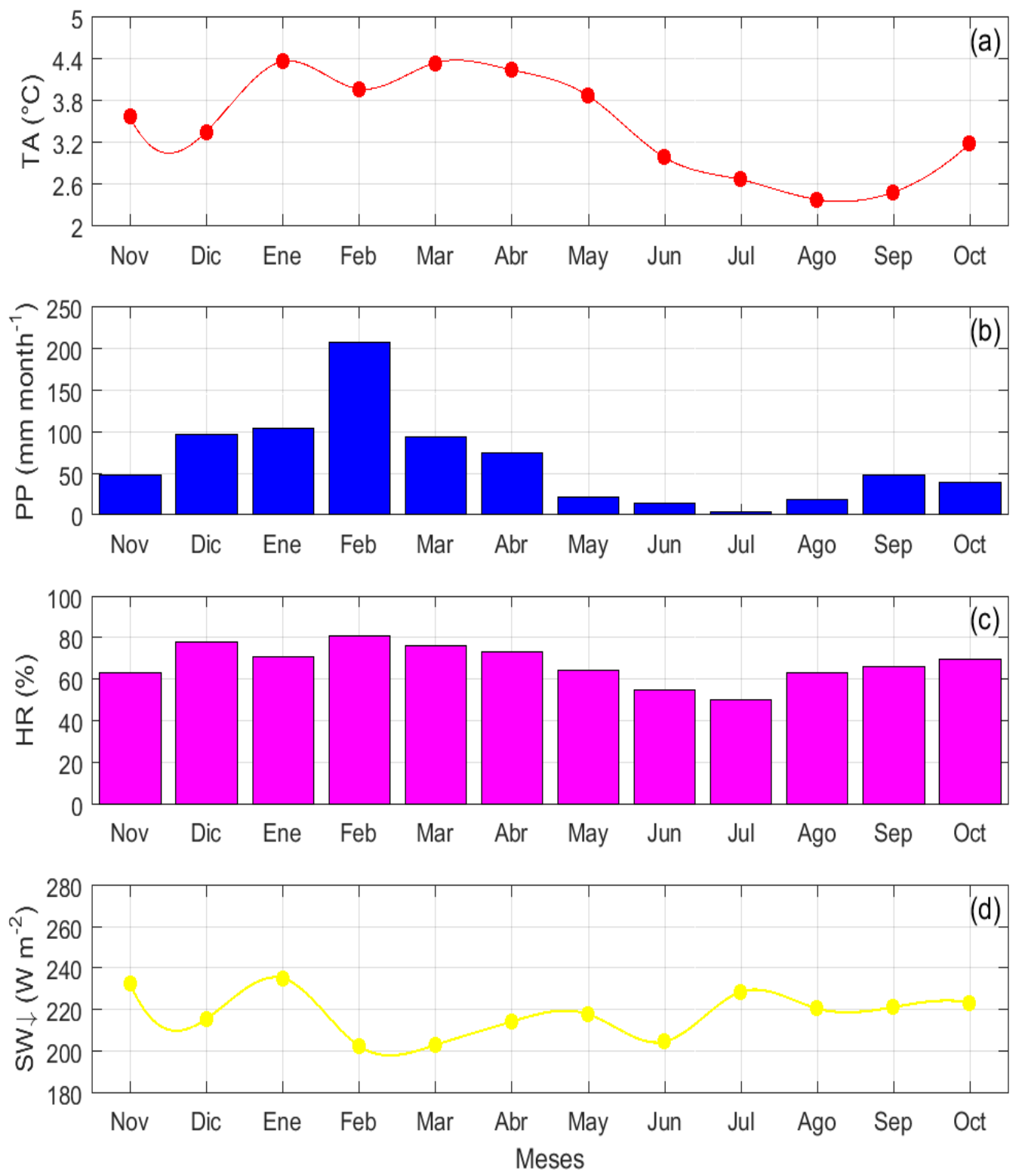

Fig.2. Variación temporal de los parámetros meteorológicos en el glaciar Huaytapallana (a) temperatura del aire, (b) precipitación, (c) humedad relativa y (d) radiación solar incidente de SW. 


\begin{tabular}{|c|c|c|c|c|c|}
\hline Fecha & $\begin{array}{c}\text { SW rad } \\
\text { incidente } \\
\left(\mathbf{W} \mathbf{m}^{2}\right)\end{array}$ & $\begin{array}{c}\text { Albedo } \\
\text { sin BC } \\
(\%)\end{array}$ & $\begin{array}{c}\text { Albedo } \\
\text { con BC } \\
(\%)\end{array}$ & $\begin{array}{c}\text { Reducción } \\
\text { de albedo } \\
\text { (\%) }\end{array}$ & $\begin{array}{c}\text { FR } \\
(\mathrm{W} \mathrm{m} 2)\end{array}$ \\
\hline $2015-11-22$ & 502.6 & 84.9 & 83.4 & 1.9 & 8.1 \\
\hline $2015-12-20$ & 634.4 & 74.7 & 73.7 & 1.4 & 6.5 \\
\hline 2016-01-17 & 362.4 & 84.9 & 84.1 & 1.1 & 3.3 \\
\hline 2016-02-21 & 719.3 & 74.7 & 74.2 & 0.7 & 3.7 \\
\hline 2016-03-20 & 454.1 & 84.9 & 82.7 & 2.7 & 10.5 \\
\hline 2016-04-17 & 561.6 & 84.9 & 84.2 & 0.9 & 4.3 \\
\hline $2016-05-22$ & 573.8 & 84.9 & 83.2 & 2.1 & 10.2 \\
\hline $2016-06-29$ & 819.7 & 75.9 & 74.5 & 2.0 & 12.5 \\
\hline $2016-07-17$ & 797.7 & 75.8 & 72.0 & 5.0 & 30.5 \\
\hline $2016-08-21$ & 531.5 & 84.9 & 82.6 & 2.9 & 12.9 \\
\hline 2016-09-25 & 664.2 & 84.9 & 82.4 & 3.0 & 17.1 \\
\hline $2016-10-23$ & 865.8 & 74.5 & 71.9 & 3.5 & 22.5 \\
\hline
\end{tabular}

\section{3.b. Descripción de los parámetros de entrada al modelo SNICAR para el glaciar Huaytapallana}

\section{3.b.1. El tipo de la radiación solar}

A partir de nuestro análisis de nubosidad, encontramos a 7 expediciones de campo con el tipo de la radiación solar difusa, mientras que 5 del tipo directa. Generalmente las condiciones difusas se encontraron en la estación de verano (diciembre - febrero) y otoño (marzo - mayo) esto es debido a que los glaciares ubicados en los Andes del centro y sur de Perú se encuentran en una región tropical bastante húmeda desde $60 \%$ hasta 71\% [35]. Además, la mayor concentración de la precipitación ocurre entre las estaciones de verano y otoño, pero también en algunos casos ocurre precipitación durante las estaciones de invierno (junio agosto) y primavera (septiembre - noviembre), las estaciones del año son consideradas para el hemisferio sur. Ha modo de ilustración presentamos en la figura 3 la cobertura de nubosidad en términos del radio efectivo de la nube para la región centro y sur de Perú. En la figura 3a presentamos para el 17 de enero y la figura 3b el 29 de junio del 2016. Se aprecia que enero gran parte de los Andes y la Amazonia están cubierto por nubes, mientras que en junio gran parte de los Andes se encuentran libre de nubes.
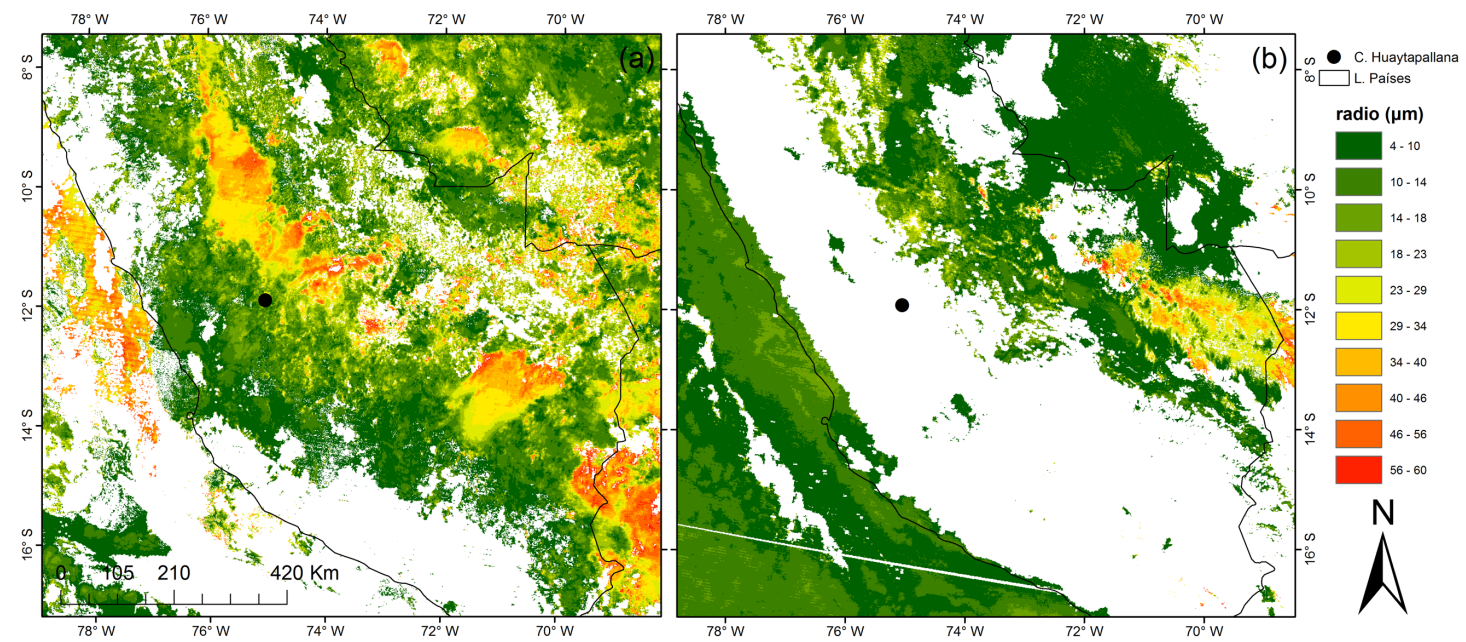

Fig.3. Cobertura de nubosidad en términos de su radio efectivo (a) para el 17 de enero y (b) para el 29 de junio del 2016 , el punto negro indica la ubicación de la Cordillera Huaytapallana. 


\section{3.b.2. Variación del SZA}

En la figura 4a se representa la variación horaria del SZA para 4 días de expedición de campo. Se observa que los valores comienzan a decrecer conforme trascurren las horas hasta llegar a los valores más bajos que se encuentran desde las 11:45 hasta 12:15 TL, pasando este horario comienza nuevamente a incrementarse. Para el 17 de enero el valor más bajo fue $8.81^{\circ}$ a las $12: 11 \mathrm{TL}$, para el $17 \mathrm{de}$ abril el valor más bajo fue $22.73^{\circ}$ a las 12:00 TL, para el 17 de julio el valor más bajo fue $32.96^{\circ} 12: 07$ TL y para el 23 de octubre el valor más bajo fue $0.23^{\circ}$ a las $11: 45 \mathrm{TL}$.

En la figura $4 \mathrm{~b}$ se representa la variación del SZA para los días de expedición de campo tomando como referencia el valor de las 11:30 TL. Se observa que la variación para 11:30 TL tiene un comportamiento bimodal. El primer pico se observa el 20 de diciembre y el segundo el 29 junio. También se observa que en los días de expedición se encontraron en otoño e invierno, el SZA se encuentra por encima de $14.5^{\circ}$. Las estaciones donde se encuentran los registros más altos de radiación solar son primavera y verano, coincidiendo con los menores valores de SZA, mientras que las estaciones con menor registro son otoño e invierno, periodo donde el SZA es mayor. Sin embargo, en las estaciones de primavera, verano y otoño la mayor parte de los días el cielo se encuentran cubierto por nubes en su totalidad, ello hace que la energía sea atenuada en la atmósfera, pero en algunos días cuando el cielo se encuentra cubierto por nubes parcialmente existe una posibilidad que las nubes contribuyan con un efecto positivo de energía conocido como dispersión múltiple.
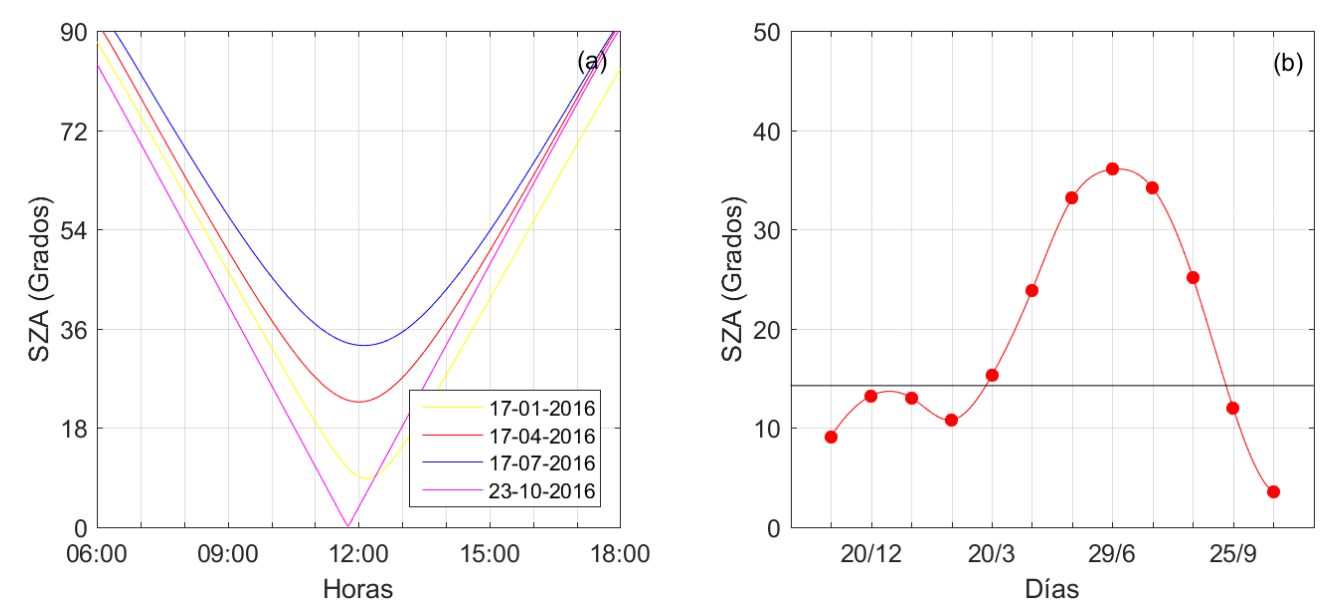

Fig.4. Variación temporal del SZA (a) horario y (b) para las 11:30 TL de los días de expedición de campo para el glaciar Huaytapallana estándar para el periodo noviembre de 2015 a octubre de 2016.

\section{3.b.3. Variación de la densidad de la nieve}

En la figura 5 se representa la variación temporal de la densidad de la capa de nieve del glaciar Huaytapallana para los días de campaña. El valor promedio fue $332 \pm 15 \mathrm{Kg} \mathrm{m}^{-3}$. Se observa que la densidad osciló entre 316-358 $\mathrm{Kg} \mathrm{m}^{-3}$. El valor más alto fue registrado el 17 de enero de 2016 mientras que el valor más bajo el 21 de junio de 2016. Esta variación está en relación directamente proporcional con la temperatura del aire de la estación Huaytapallana. Durante los meses más fríos se encontraron valores más bajos mientras que los meses más cálidos fueron sustancialmente valoras más altos. Valores similares fueron reportados en otros estudios para otros glaciares en el mundo [36], [37]. Sobota (2011) reportó que la densidad de la nieve en los glaciares de Svalbard (lat. $-10^{\circ}$ a $-35^{\circ}$; lon. $76^{\circ}$ a $81^{\circ}$ ) oscila entre $346-378 \mathrm{~kg}$ $\mathrm{m}^{-3}$. 


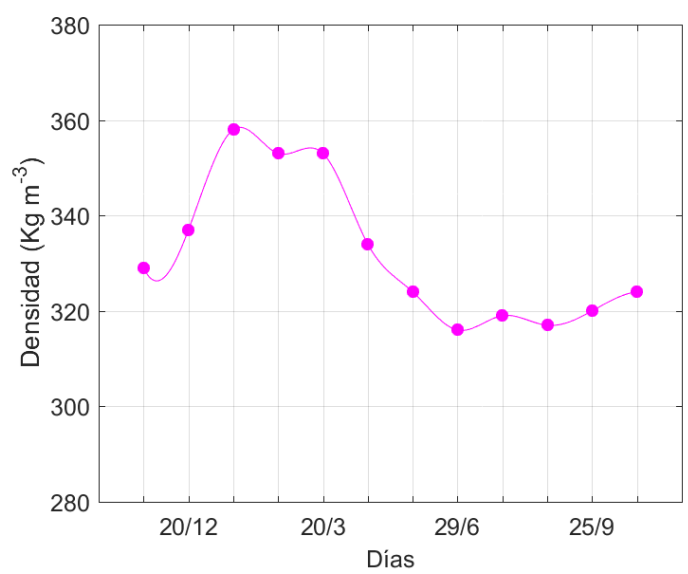

Fig.5. Variación temporal de la densidad de la capa de nieve en el glaciar Huaytapallana para los días de expedición de campo para el periodo noviembre de 2015 a octubre de 2016.

\section{3.c. Variación del CNe}

En la figura 6 se observa la variación temporal de la masa del CNe en la capa superficial de 0 a $2 \mathrm{~cm}$ de profundidad. El promedio del CNe para todo el periodo de estudios fue $31.1 \pm 22.2 \mathrm{ppb}$. Los valores oscilaron entre 4-84 ppb que son similares a lo reportado en otros estudios realizados como en la Cordillera Blanca [24]; en el Monte Nyainqentanglha [6]; y en el Norte América central [39], con intervalos entre 2-80 ppb; 4$70 \mathrm{ppb}$; y 5-70 ppb, respectivamente. Sin embargo, estos valores son menores a los reportados en los glaciares ubicados al norte de China [40], con valores entre 117-1120 ppb. Se debe resaltar que el 20 de marzo de 2016 la masa del CNe se incrementó en 13 veces respecto al mes anterior y en 5 veces más respecto al mes siguiente. Al realizar la prueba estadística no paramétrica de suma de rangos de Wilcoxon encontramos que existen diferencias significativas de la masa del CNe para el periodo lluvioso (septiembre - mayo) y seco (junio - agosto) con un p-valor de $2.16 \times 10^{-4}$. La masa del CNe aumentó con el tiempo desde mayo hasta octubre de 2016 en la temporada seca (junio - agosto). Este incremento probablemente está influenciado por el aumento de los incendios de vegetación abierta que ocurren en América del Sur como lo reporta Vasconcelos et al. (2013).

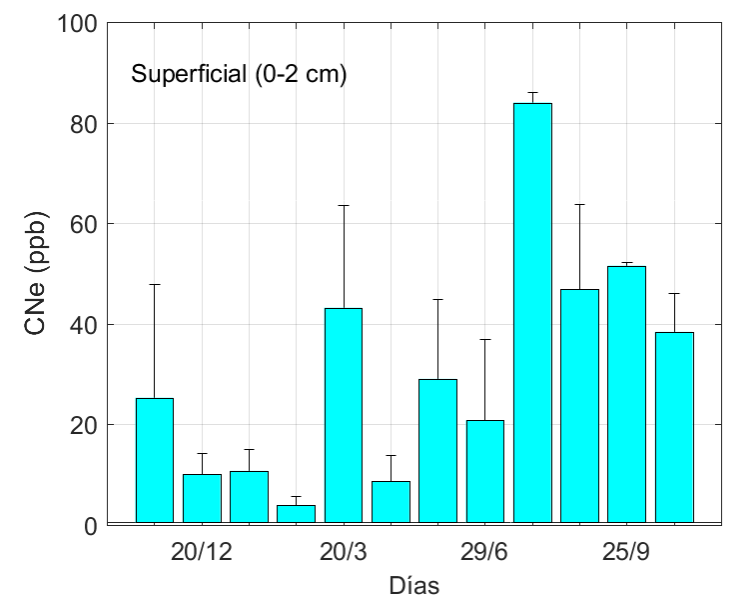

Fig.6. Variación temporal de la masa del CNe en capa superficial (0-2 cm de profundidad) en la nieve del glaciar Huaytapallana, las barras representan la promedio y las barras de error las desviación estándar para el periodo noviembre de 2015 a octubre de 2016.

\section{3.d. Efecto en el albedo y el FR}

En la figura 7a se observa la reducción del albedo de la superficie del glaciar como consecuencia de la deposición del $\mathrm{CNe}$ en la nieve. La reducción del albedo osciló entre 0.6-5.0\%, valores similares a los repostados por otros estudios [9], donde señala que el rango de reducción posible en el albedo, debido a deposición del $\mathrm{CN}$ en la nieve de los glaciares del Himalaya, se encuentra entre 4-5\%. Otros estudios reportan que el albedo en la nieve del Himalaya se redujo entre 2-6\% [42], valores menores a los reportados 
por Flanner et al. (2007) donde se reporta que un incremento de $500 \mathrm{ppb}$ del CN en la nieve disminuye el albedo en 10\%. Por su parte Dumont et al. (2014) menciona que el albedo de la nieve en Groenlandia se redujo en un 13\% como consecuencia de la deposición de CN. En el presente estudio los valores más altos de reducción de albedo se observan durante la temporada seca (junio-agosto).

La deposición de las PALs reduce el albedo de la superficie y como consecuencia mayor energía queda retenida, favoreciendo la fusión glaciar, que ocasiona un retroceso más acelerado. En este estudio se demuestra que el FR instantáneo, como consecuencia de la deposición del CNe en la nieve del glaciar Huaytapallana (figura $7 \mathrm{~b}$ ), contribuye en promedio, para todo el periodo de estudios, $11.8 \pm 7.8 \mathrm{~W} \mathrm{~m}^{-2}$, que es el doble de lo reportado por Ming et al. (2013) con un FR del CN promedio de $6 \mathrm{~W} \mathrm{~m}^{-2}$ (aproximadamente $5 \%$ del forzamiento total) en los glaciares de Asia alta. Los valores del FR oscilaron entre 3-30 W m-2. El estudio de Flanner et al. (2007), estimó que el FR debido a la presencia del CN sobre algunos glaciares de la Meseta Tibetana, excedió los $20 \mathrm{~W} \mathrm{~m}^{-2}$. Por su parte Sterle et al. (2013) reportan que la deposición del CN en la nieve conlleva a un FR que oscila entre 20-40 W m² durante abril y mayo en Sierra Nevada. En la Meseta Tibetana la deposición del CN contribuye con el FR entre 1-141 W m-2 [43], pero estos valores son más bajos que de los reportados por Niu et al. (2017) quien reporta valores de FR entre 76-147 W m² para la misma región.

Al analizar los valores del FR por estaciones del año, se observa un incremento del 157\% durante el invierno (junio - agosto) y 134\% para la primavera (septiembre - noviembre), mientras que estos valores se reducen para el verano (diciembre - febrero) en 62\% y para el otoño (marzo - mayo) en 29\%, respecto al promedio general. Finalmente, haciendo la prueba estadística de Spearman con un nivel de significancia 0.05 se observa una fuerte correlación positiva entre el CNe y el FR $\left(F R=0.31 \times C N e+1.98, R^{2}=0.87, p-\right.$ valor $=4.43 \times 10^{-4}$ ). Por lo tanto, el impacto de la deposición de $\mathrm{CNe}$ en el retroceso del glaciar Huaytapallana es un factor importante y debe ser considerado en los modelos hidrológicos de predicción futura de disponibilidad de agua.
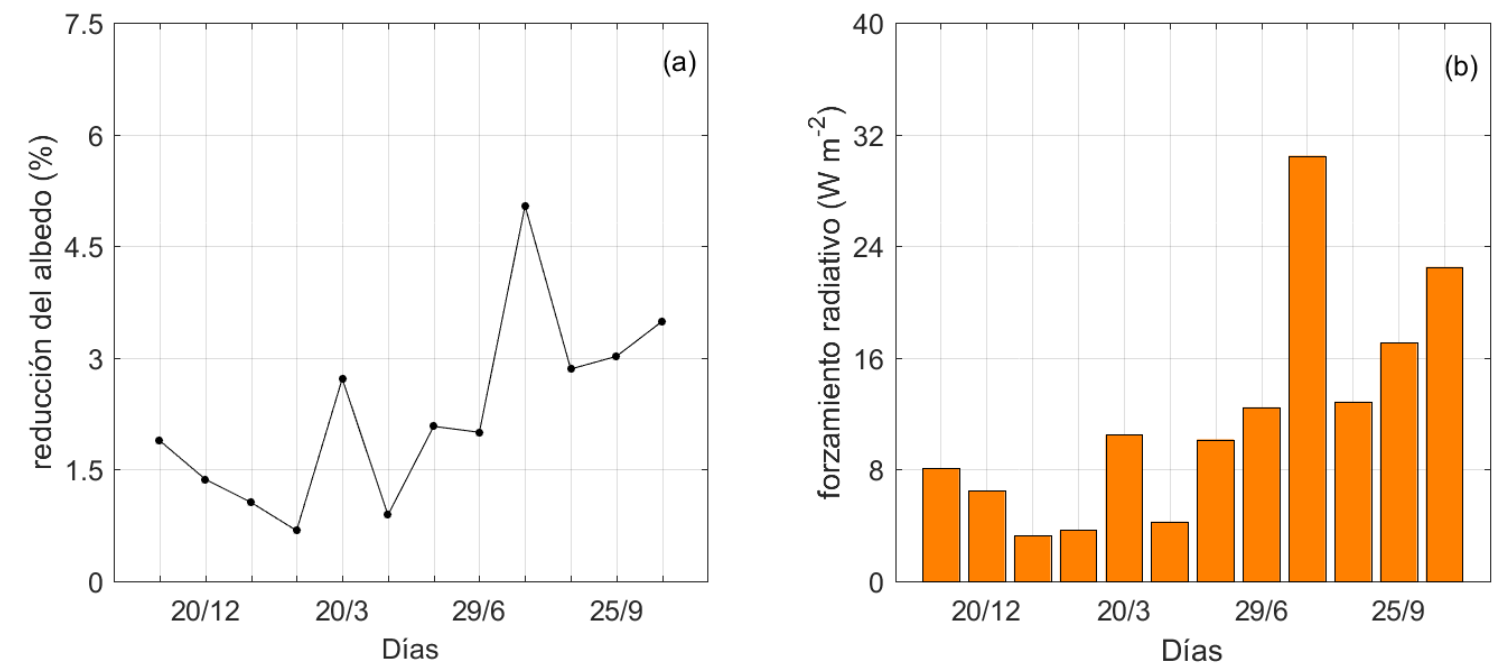

Fig.7. Reducción del albedo de la superficie (a) y del FR (b) por deposición de CNe en la nieve del glaciar Huaytapallana estándar para el periodo noviembre de 2015 a octubre de 2016.

\section{Conclusiones}

Este estudio se basó en las primeras mediciones de campo del contenido del CNe en la nieve del glaciar

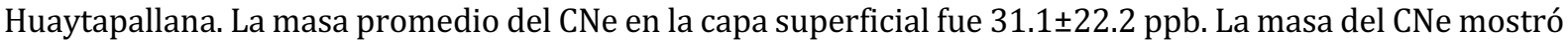
una gran variación temporal con valores que oscilaron entre 4-84 ppb, con un incremento durante las estaciones de invierno y primavera. La reducción del albedo de la superficie del glaciar, inducida por la presencia del CNe, osciló entre 0.6-5.0\%. Se estimó que el FR del CNe en la nieve del glaciar Huaytapallana fue $11.8 \pm 7.8 \mathrm{~W} \mathrm{~m}^{-2}$. Pero si se analiza por estaciones este valor se incrementa significativamente para invierno en un $157 \%$ y para primavera en un $134 \%$, respecto al promedio general. Finalmente, se observó que existe una correlación positiva fuerte entre el CNe y el FR $\left(R^{2}=0.87\right)$, lo que sugiere que la presencia 
del $\mathrm{CNe}$ en el glaciar Huaytapallana es un factor importante en los procesos de desglaciación y debe ser considerado en los modelos hidrológicos de predicción futura de disponibilidad de agua.

\section{Agradecimientos}

Se agradece al Dr. Mark Flanner por proporcionarnos el código fuente del modelo SNICAR y por su orientación en su uso. Al Dr. Steven Platnick por orientarnos con la metodología para determinar el tipo de radiación solar incidente. A la Agencia de los Estados Unidos para el Desarrollo Internacional (USAID, por sus siglas en inglés) por el financiamiento de esta investigación a través del Proyecto PEER-Aerosoles "Impact of transboundary biomass burning pollution transport over the Central Andes of Peru" del Partnerships for Enhanced Engagement in Research (PEER) Science Program from National Academy of Sciences (NAS). Al Servicio Nacional de Meteorología e Hidrología (SENAMHI) del Perú por los datos meteorológicos proporcionados de la estación Huaytapallana. Así mismo, agradecemos a los revisores anónimos por hacer comentarios útiles sobre el artículo. 